\title{
Knockdown of ILK inhibits glioma development via upregulation of E-cadherin and downregulation of cyclin D1
}

\author{
KEBIN ZHENG, GUANGYI WANG, CHUNHUI LI, XIAOSONG SHAN and HAIPENG LIU \\ Department of Neurosurgery, Affiliated Hospital of Hebei University, Baoding, Hebei 071000, P.R. China
}

Received January 26, 2015; Accepted March 19, 2015

DOI: $10.3892 /$ or.2015.3983

\begin{abstract}
Integrin-linked kinase (ILK) is a highly conserved serine-threonine protein kinase that interacts with cytoplasmic domains of integrin subunits in tumor tissues. However, the relationship between gliomas and ILK is elusive. The present study aimed to investigate the role of ILK in a human glioma cell line (U251). ILK stable expressing vector, U251ILKPGFP-V-RS-shRNA, was established and named as U251-si. The empty-PGFP-V-RS-shRNA (U251-N) was employed as the control. Quantitative real-time PCR and western blot analysis were used to detect ILK and E-cadherin mRNA and protein expression, respectively. Cell cycle analysis was employed to examine the cell cycle distribution. Cell migration was detected using a wound healing assay, and cell invasion was detected using a Transwell invasion assay. Tumor size and weight were also examined. The results indicated that ILK was expressed at a lower level at both the mRNA and protein levels in the U251-si group compared with the U251-N group ( $\mathrm{p}<0.01)$. ILK knockdown suppressed cell proliferation of the glioma cells. Knockdown of ILK reduced the migratory and invasive potentials of the glioma cells. Inhibition of ILK expression upregulated E-cadherin and downregulated cyclin D1 in the glioma cells compared to the U251-N group $(\mathrm{p}<0.05)$. Knockdown of ILK in the U251 cells attenuated the ability of U251 cells to form tumors in nude mice and impaired glioma cell in vivo tumorigenicity. In conclusion, knockdown of ILK inhibits glioma cell migration, invasion and proliferation through upregulation of E-cadherin and downregulation of cyclin D1. Our results suggest that ILK may serve as a promising therapeutic target for glioma.
\end{abstract}

\section{Introduction}

Gliomas are the most common primary malignant brain tumors that originate from neuroepithelial tissue (1-3). They

Correspondence to: Dr Chunhui Li, Department of Neurosurgery, Affiliated Hospital of Hebei University, Baoding, Hebei 071000, P.R. China

E-mail: lichunhuineuro@yeah.net

Key words: glioma, integrin-linked kinase, E-cadherin, cyclin D1, proliferation are associated with a late diagnosis, poor prognosis, and high mortality rate. Despite aggressive multimodal therapy with maximal resection followed by the latest available therapeutic interventions, these tumors still have a dismal prognosis with a median survival rate of only 2 years and a 3 -year survival rate of only $10 \%$ (4). There is currently no effective treatment for glioma. Glioma is a highly complicated and heterogeneous tumor condition that results from the aberrant activation of numerous important signaling pathways (5). Understanding the molecular mechanisms of glioma is of great importance in the search for a curative therapy.

Integrin-linked kinase (ILK) is a highly conserved serinethreonine protein kinase that interacts with the cytoplasmic domains of integrin subunits. Since its initial discovery in 1996, ILK has emerged as a key regulator of the phosphatidylinositol 3-kinase (PI3-K) signaling pathway, which activates protein kinase $\mathrm{B}(\mathrm{PKB}) / \mathrm{Akt}$ activity and inhibits cyclin D1, thereby inhibiting proliferation of different types of cancer cells (6). Overexpression of ILK has been shown to promote cell migration and invasion (7-11). In particular, inhibition of E-cadherin transcription is thought to be a key mechanism by which ILK promotes cancer cell invasion and migration.

In the present study, we investigated the role of ILK in a human glioma cell line (U251). Our results demonstrated an important and essential role of ILK in two key aspects of glioma, including apoptosis and the migration and invasion process.

\section{Materials and methods}

Cell culture. The human glioma cell line U251 was obtained from the Cell Bank of the Chinese Academy of Sciences (Shanghai, China) and cultured in Dulbecco's modified Eagle's medium (DMEM; Gibco, Grand Island, NY, USA) supplemented with $10 \%$ fetal bovine serum (FBS; HyClone, Logan, UT, USA) and penicillin/streptomycin (100 U/ml and $100 \mathrm{mg} / \mathrm{ml}$ ) at $37^{\circ} \mathrm{C}$ in a $5 \% \mathrm{CO}_{2}$ humidified atmosphere.

Plasmid construction and stable transfection. To generate a ILK stable expressing cell line, U251 cells were seeded into 6-well plates at a density of $1 \times 10^{6}$ cells/well. When U251 cell confluency reached $75-80 \%$, the U251 cells were transfected with different PGFP-V-RS-shRNA constructs using Lipofectamine ${ }^{\mathrm{TM}} 2000$ (Invitrogen, Carlsbad, CA, USA), according to the manufacturer's protocol. One clone 
was transfected with ILK-PGFP-V-RS-shRNA and the other was transfected with empty-PGFP-V-RS-shRNA. At $48 \mathrm{~h}$ post-transfection, stable clones were selected with puromycin $(8 \mu \mathrm{g} / \mathrm{ml})$ for 3 weeks. Then the stable clones were screened for GFP expression by a fluorescence microscope and assayed for ILK expression by quantitative reverse transcriptase-PCR (qRTPCR) and western blot analysis. ILK-PGFP-V-RS-shRNA was named U251-si and empty-PGFP-V-RS-shRNA was named U251-N. The control cell line named U251 was not transfected.

Quantitative real-time PCR. Total RNA was extracted from cells using TRIzol reagent (Life Technologies, Rockville, MD, USA) according to the manufacturer's instructions. First-strand complementary DNA (cDNA) was synthesized from $1 \mu \mathrm{g}$ of total RNA using the Prime Script First Strand cDNA Synthesis kit (Takara, Dalian, China). Quantitative real-time PCR reactions were carried out with cDNA $(1 \mu \mathrm{l})$ and the SYBR-Green Master Mix (Takara) on a Real-Time Quantitative Thermal Block (Biometra, Göttingen, Germany). The sequences of primers are listed in Table I. $\beta$-actin served as an internal control. The specificity of the PCR was confirmed by melting curve analysis. Data were treated using the comparative threshold cycle (CT) method (12).

Western blot analysis. Proteins were extracted with a buffer composed of $50 \mathrm{mM}$ Tris- $\mathrm{HCl}, \mathrm{pH} \mathrm{7.5,0.1 \%}$ Triton X-100, ethylenediaminetetraacetic acid (EDTA) $5 \mathrm{mM}$, and a cocktail of protease inhibitors. The following primary antibodies were used: anti-E-cadherin, anti-ILK, anti-Bcl-2, anti-cyclin D1, and anti-actin (Santa Cruz Biotechnology, Inc., Santa Cruz, CA, USA).

Cell cycle analysis. Cells were cultured with or without drug treatment for $24 \mathrm{~h}$ and subsequently analyzed. Control cells were treated with the vehicle control PTE [PEG300/ethanol/ Tween 80/citrate (63:29:7.8:0.2, w/v/w/w)]. Cells were harvested, fixed with cold $70 \%$ ethanol, and stored overnight at $-20^{\circ} \mathrm{C}$, followed by staining with propidium iodide staining buffer $(1 \mathrm{mg} / \mathrm{ml}$ RNase A, $0.1 \%$ Triton X-100, $50 \mu \mathrm{g} / \mathrm{ml}$ propidium iodide in PBS).

Stained samples were analyzed by flow cytometry with a FACSCalibur (Becton-Dickinson, San Jose, CA, USA) or with WinMDI 2.9 freeware to determine cell cycle distribution. The percentage of cells in each cell cycle phase was calculated relative to the total cells in the G1-G0, S and G2-M phases after prior exclusion of pre-G1-G0 events.

Wound healing assay. Cells were plated in 6-well plates at a density of $1 \times 10^{5}$ cells per well and grown to approximately $80 \%$ confluency. The monolayer was scraped with a sterile $200-\mu 1$ pipette tip after removal of the culture medium. Subsequently, the culture was washed twice with serum-free medium. After that, cells were maintained in DMEM, the scratched areas were photographed at 0,12 and $24 \mathrm{~h}$ after wounding using computer-assisted microscopy. Cell migration was calculated as the percentage of cell coverage to the initial cell-free zone.

Transwell invasion assay. Cell invasion was evaluated using a Transwell chamber (Corning Costar, Cambridge, MA, USA) equipped with a Matrigel-coated filter membrane $(8-\mu \mathrm{m}$
Table I. Sequences of the primers.

\begin{tabular}{ll}
\hline Genes & \multicolumn{1}{c}{ Sequences } \\
\hline ILK & F: 5'-TGAAGACACAAACAGACG-3' \\
& R: 5'-TCAAGGATAGGCACAATC-3' \\
E-cadherin & F: 5'-ATGCCGCCATCGCTTACAC-3' \\
& R: 5'-CGACGTTAGCCTCGTTCTCA-3' \\
Cyclin D1 & F: 5'-ACCTGAGGAGCCCCAACAA-3' \\
& R: 5'-TCTGCTCCTGGCAGGCC-3' \\
$\beta$-actin & F: 5'-GTTGCCCTGAGGCTCTTTTCC A-3' \\
& R: 5'-ACCACCAGACAGCACTGTGTTG-3'
\end{tabular}

$\mathrm{F}$, forward primer; $\mathrm{R}$, reverse primer.

pores). Briefly, the filters were pre-coated with $0.5 \mu \mathrm{g}$ basement membrane proteins (Matrigel; BD Biosciences, San Jose, CA, USA) and allowed to dry overnight at room temperature. Cells in FBS-free medium were seeded in the upper chambers, and the lower wells contained 10\% FBS medium. After incubation at $37^{\circ} \mathrm{C}$ for $24 \mathrm{~h}$, non-migratory cells on the upper side of the insert were removed with a cotton swab. Cells that had passed through the filter were fixed in methanol and stained with hematoxylin. For quantification, six randomly selected fields on the lower side of the insert were photographed using computer-assisted microscopy.

Statistical analysis. Statistical analyses were performed with SPSS version 13.0 (SPSS, Inc., Chicago, IL, USA). Data are expressed as means \pm SD. Differences between groups were analyzed by one-way ANOVA. A p-value $<0.05$ was considered statistically significant.

\section{Results}

Stable downregulation of ILK in the U251 cells. One effective way to understand the physiological role of ILK in glioma is to inhibit the expression of endogenous ILK in glioma cell line models. The first efficient knockdown of ILK expression was achieved by the successful delivery and expression of short-hairpin (sh)RNA targeting ILK in the U251 cells. We detected expression of ILK in the U251 cells using immunofluorescence staining and western blot analysis. Immunofluorescence staining showed that ILK was mainly localized at the cell membrane and that immune intensity was obviously decreased in the the ILK-downregulated cells as compared with the control cells. ILK demonstrated low expression at both the mRNA and protein levels in the ILK-knockdown stable transfected clones compared with the empty-vector transfected cells (Fig. 1, p<0.01).

Knockdown of ILK suppresses glioma cell growth. To determine the effect of ILK expression on glioma cell growth, proliferation curves of the ILK-knockdown stable clones and their corresponding non-targeted controls were compared. As shown in Fig. 2A, similar proliferation rates were observed for U251, U251-N, and U251-si cells during the first $48 \mathrm{~h}$. 


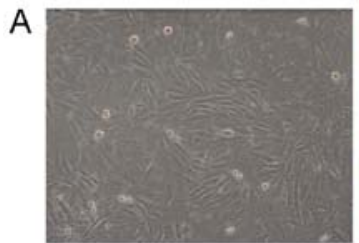

U251

B

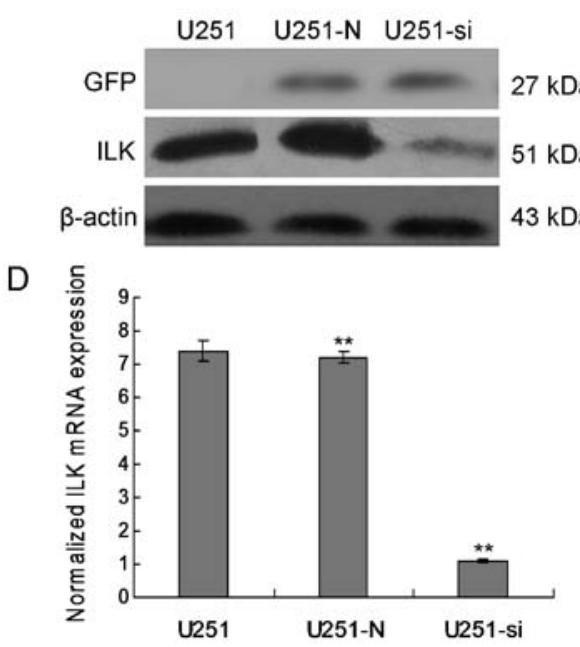

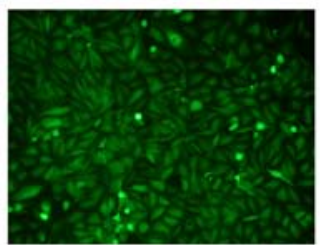

U251-si

C

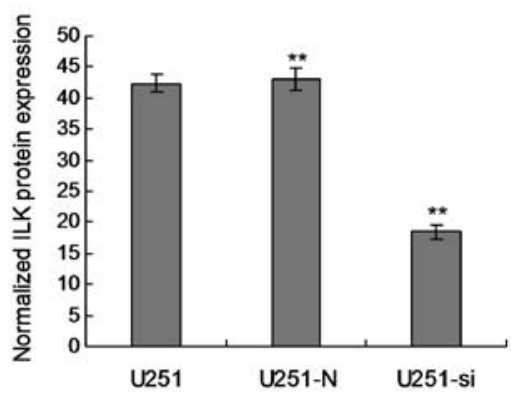

Figure 1. ILK expression in stably transfected U251 cells. (A) Immunofluorescence staining of ILK on the surface of the stable cells. (B) Western blot analysis of ILK expression in the transfected cells. The blots shown are representative of three independent experiments. (C) Quantitative analysis of ILK protein expression. The band density values were calculated as a ratio to $\beta$-actin. Data are mean \pm SD. (D) Quantification of ILK mRNA from three independent real-time PCR experiments, normalized to the expression of $\beta$-actin. ${ }^{* *} \mathrm{p}<0.01$ compared with the empty vector-transfected cells.

A

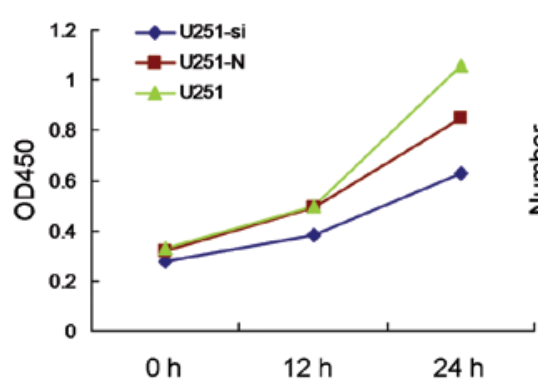

C

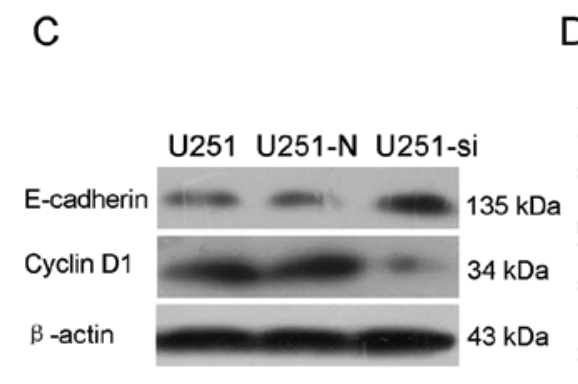

B

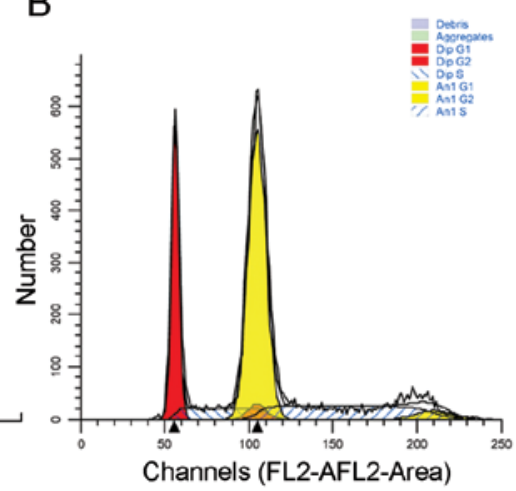

U251

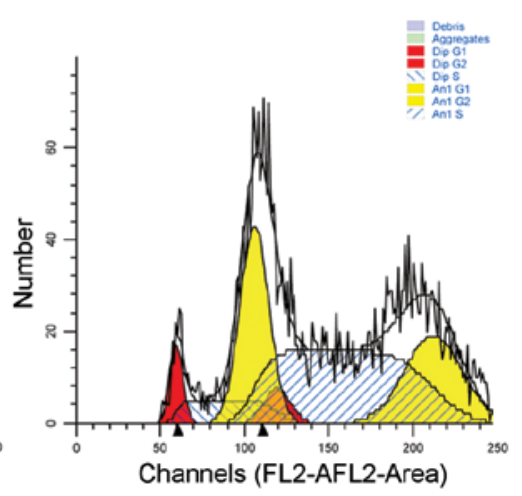

U251-si

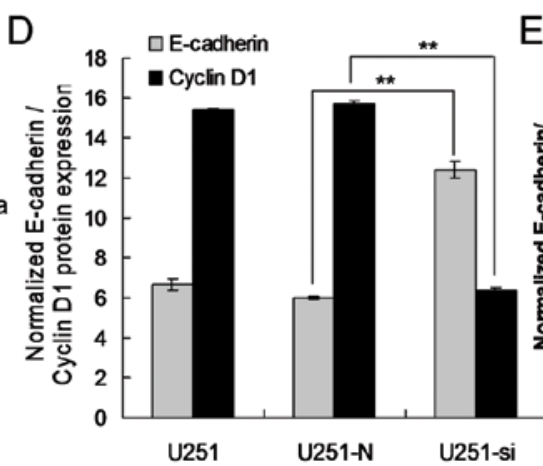

E

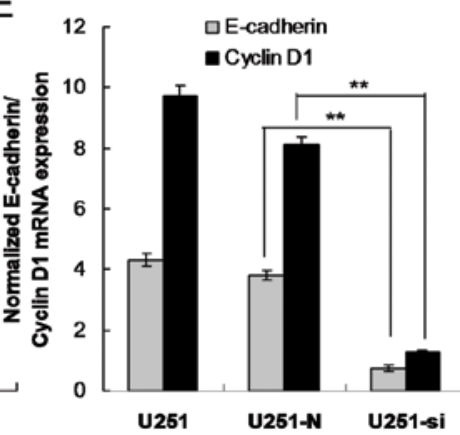

Figure 2. Stable downregulation of ILK decreases glioma U251 cell proliferation, migration, and invasion capacities in vitro. (A) MTT proliferation assay. U251 cells were seeded into 96-well plates and incubated for various time intervals. Experiments were performed in triplicate. (B) Flow cytometry showing cell cycle arrest in the G2/M phase. (C) Western blot analysis of E-cadherin and cyclin D1 in stable cells. Representative blots are shown. Protein size is expressed in $\mathrm{kDa}$. (D) Densitometric quantification data are expressed as target proteins to $\beta$-actin. (E) Real-time PCR analysis of E-cadherin and cyclin D1 mRNA levels in the stable cells. Gene expression was normalized to $\beta$-actin and expressed as relative change. ${ }^{* * *} \mathrm{p}<0.01$ compared with the empty vector-transfected cells. 

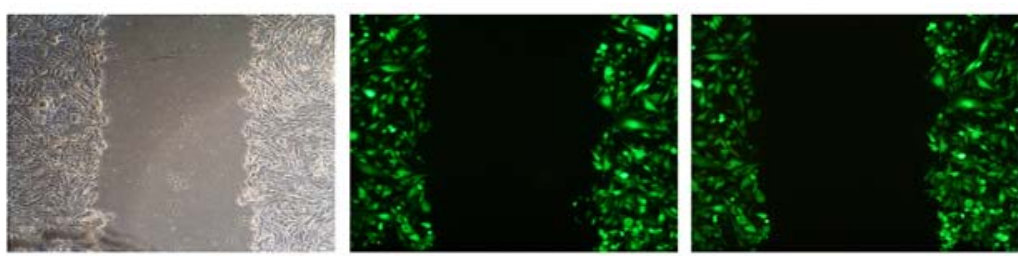

$12 \mathrm{~h}$
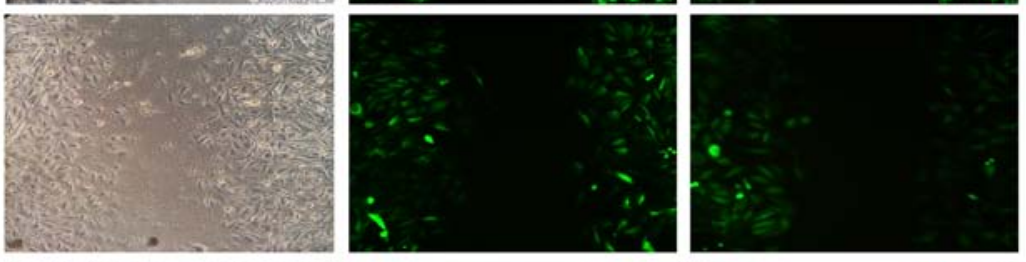

$24 \mathrm{~h}$
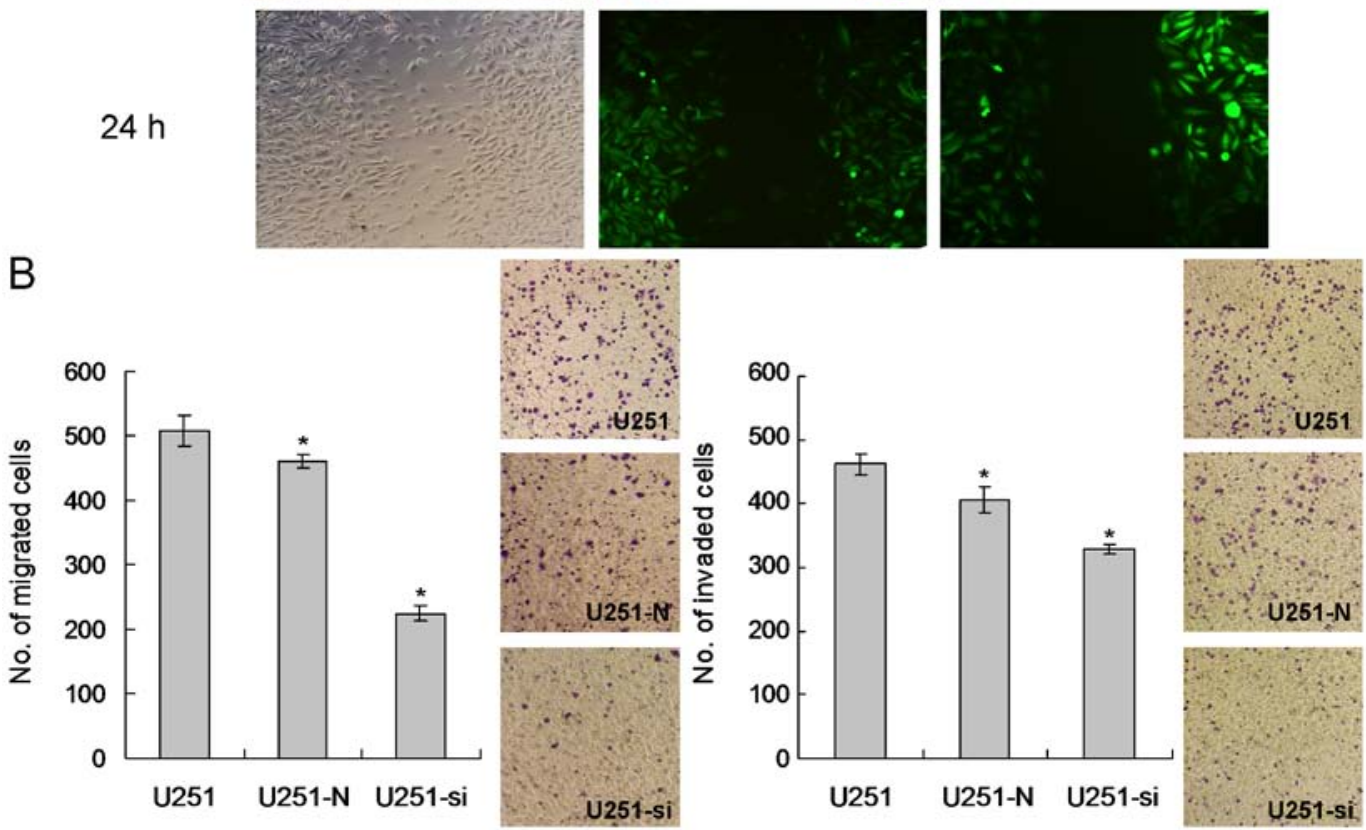

Figure 3. ILK knockdown inhibits cell migration and invasion. (A) Wound healing was examined in the stable cells at 0,12 and 24 h after wound formation Representative images from three experiments are shown. Cell migration was calculated and expressed as the percentage of cell coverage to the initial cell-free zone. Values are presented as mean \pm SD of three separate experiments. (B) Representative images of the invaded cells (stained with hematoxylin) selected from three experiments are shown. Migrated cells were counted under a microscope. The graph shows the mean \pm SD. ${ }^{* *} \mathrm{p}<0.01$ compared with the empty vector-transfected cells. Scale bars represent $100 \mu \mathrm{m}$.

However, starting from $72 \mathrm{~h}$, a significant difference in cell growth was observed between the ILK-knockdown clones and the non-targeted controls. U251-N was found to have a higher proliferation rate when compared to the U251-si cells. This trend persisted until the last day of the proliferation assay. As shown in Fig. 2B, flow cytometry showed that the cell cycle was arrested in the $\mathrm{G} 2 / \mathrm{M}$ phase. These results indicate that ILK-knockdown suppressed the cell proliferation of glioma cells.

Knockdown of ILK reduces the migratory and invasive potentials of glioma cells. To assess the migratory ability of the ILK-knockdown stable clones, wound healing and a Transwell migration chamber were employed. Differences in wound-closure ability were obvious in the ILK-knockdown stable clones. Wounds in the U251-N control clones were almost closed in $24 \mathrm{~h}$, yet the wounds in the U251-si stable clones were still clearly observed (Fig. 3A). The Transwell migration assay revealed that the number of migrated cells in the U251-N control clones was significantly higher when compared to the U251-si stable clones (Fig. 3B). Cell invasiveness of the
ILK-knockdown stable clones was assessed by seeding the cells onto a Matrigel-coated invasion chamber. More invaded cells were observed in the U251-N control while significantly fewer cells were able to invade through the Matrigel in the U251-si stable clones. Collectively, these results indicated that knockdown of ILK suppressed cell invasion.

Inhibition of ILK expression upregulates E-cadherin and downregulates cyclin D1 in the glioma cells. To investigate whether E-cadherin contributes to the cell migration and invasion induced by knockdown of ILK, we detected expression of E-cadherin in the U251 cells using immunofluorescence staining, real-time PCR and western blot analysis. Immunofluorescence staining showed that exogenous E-cadherin was mainly localized at the cell-cell contacts and that immune intensity was obviously increased in the U251-si cells as compared with the U251-N cells (Fig. 4). Meanwhile, western blot analysis consistently showed that the protein expression level of E-cadherin was significantly increased in the U251-si cells as compared to the U251-N cells (Fig. 2C and D, p<0.01). The real-time PCR data 


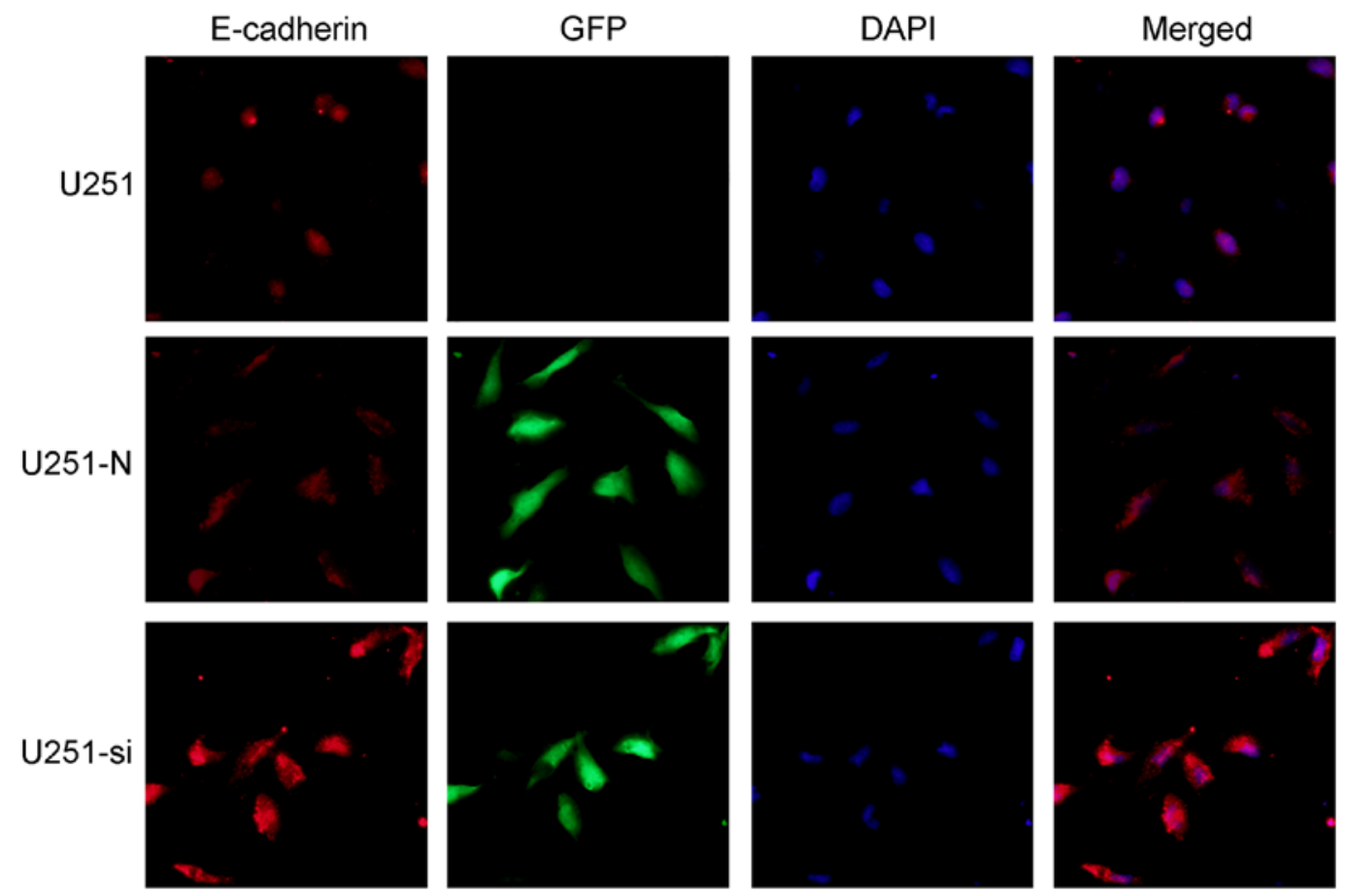

Figure 4. Immunofluorescence staining of E-cadherin on the surface of the stable cells. Cells were fixed with acetone. E-cadherin on the cell surface was stained with a rabbit anti-E-cadherin antibody and a FITC-labeled secondary antibody. E-cadherin immunofluorescence (red) was imaged along with the nuclei stained with DAPI (blue). Scale bars, $100 \mu \mathrm{m}$.

A

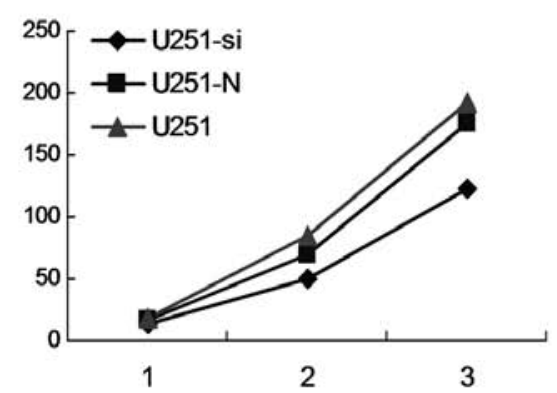

C

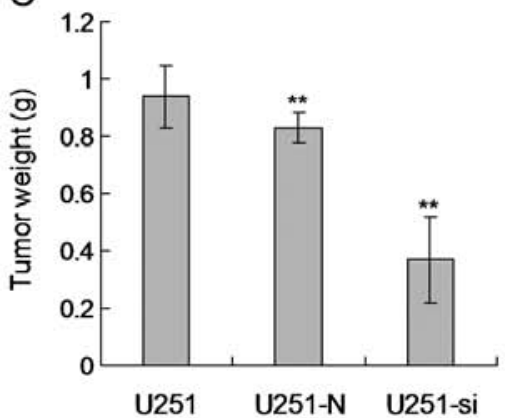

B
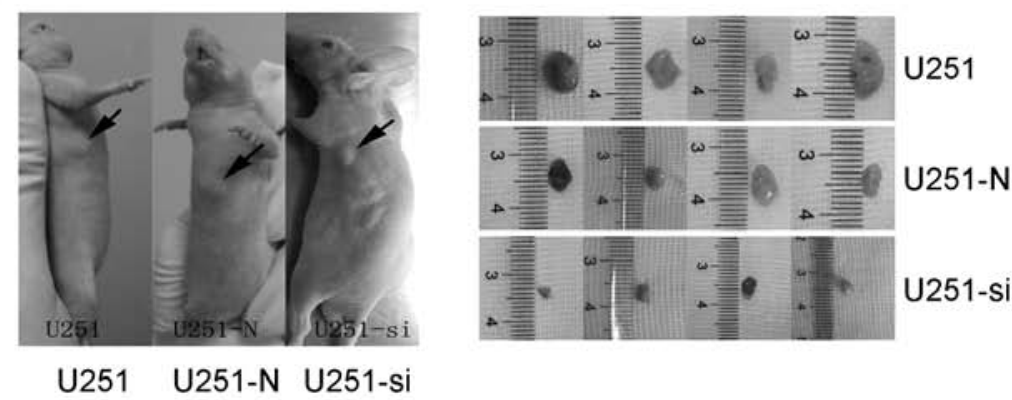

D

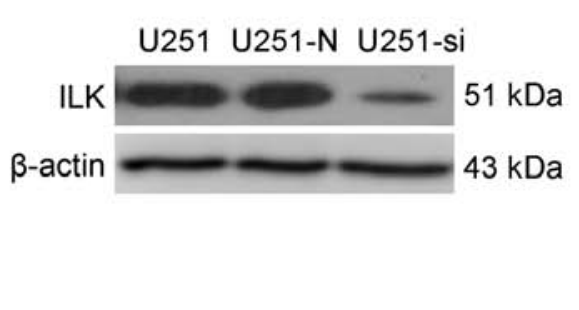

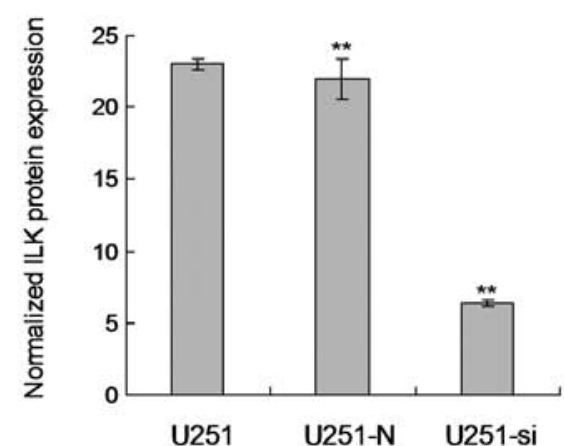

Figure 5. ILK knockdown suppresses in vivo tumorigenicity of U251 cells. (A) ILK-knockdown clones were injected subcutaneously into nude mice with $5 \times 10^{4}$ cells/site. Tumor volume was measured every week by the following formula: $\mathrm{V}=1 / 2 \mathrm{x}$ a (length) $\mathrm{x} \mathrm{b}$ (width). (B) Mice were sacrificed at the third week after subcutaneous injection. (C) Tumors were harvested, photographed and weighed. (D) Protein was extracted from the excised tumors and analyzed for ILK expression by western blot analysis. ${ }^{* *} \mathrm{p}<0.05$.

were also in direct agreement with the results of the western blot analysis (Fig. 2E, p <0.01). Our data indicated that down- regulation of E-cadherin was involved in the ILK-induced cell migration and invasion. 
As shown in Fig. 2C, the protein level of cyclin D1 was significantly reduced in the U251-si cells. Additionally, such treatment resulted in a marked reduction in mRNA expression in the U251-si cells (Fig. 2E). Collectively, these results suggest that upregulation of cyclin D1 is involved in ILK-induced cell proliferation.

Knockdown of ILK impairs glioma cell in vivo tumorigenicity. U251-si stable clones were subjected to nude mouse injection. After subcutaneous injection of the cells into the nude mice, tumor growth was monitored carefully and the size of the tumors formed was measured weekly. Starting from week 3 , larger tumors were formed in the mice injected with the NT control clones while smaller tumors were formed from the stable clones (Fig. 5A). At week 3, the animals injected with U251-si were sacrificed due to oversized tumors (Fig. 5B). Tumors were then harvested from the mice, photographed and weighed. Tumors formed from the U251-N control clones were significantly larger than the tumors from the U251-si stable clones. This trend was also reflected in the weight of the tumors where the average tumor weight from the U251-N control clones was significantly higher than that from the U251-si clones (Fig. 5C). This result demonstrated that suppression of ILK in U251 cells attenuated the ability of U251 cells to form tumors in nude mice. In addition, protein was extracted from the excised tumors and analyzed for ILK expression by western blot analysis. ILK protein expression was decreased in the tumor tissues of the mice injected with the U251-si clones (Fig. 5D).

\section{Discussion}

ILK is widely expressed in several types of human cancers including glioma and has been positively correlated with tumor growth and closely related to tumor apoptosis, proliferation, invasion and migration $(13,14)$. ILK is a ubiquitously expressed serine/threonine protein kinase that binds directly to the cytoplasmic domains of $\beta 1$ and $\beta 3$ integrins and has been shown to have clinical/prognostic and functional significance in various human cancers.

In spite of the potential significance of ILK in glioma, the functional role of ILK and elucidation of its associated pathways in glioma have not been clearly defined. To understand the functions of ILK, endogenous ILK expression in glioma cell lines was silenced by shRNA. ILK-knockdown stable clones displayed suppressed cell proliferation and anchorage-independent growth. Additionally, motility and invasiveness of the cells were largely impeded upon ILK depletion. Glioma cells with suppressed ILK expression displayed an inhibited ability to form tumors in nude mice. All of these studies corroborate our findings that ILK exerts an oncogenic effect on glioma cell lines.

Recently, ILK has been shown to induce E-cadherin shedding and to promote cell migration and invasion in lung cancer cells (15). Thus, we sought to study the effects of ILK on E-cadherin expression. E-cadherin is a transmembrane glycoprotein that mediates calcium-dependent intracellular adhesion in normal epithelial cells. Selective loss of E-cadherin function or expression has been linked with cancer progression and metastasis. In the present study, our results showed that inhibition of ILK increased E-cadherin expression at the mRNA and protein levels and decreased cell migratory and invasive abilities in the glioma cells. Our results strongly suggest that ILK suppresses E-cadherin expression to inhibit invasion and migration. However, the mechanisms underlying tumor metastasis by ILK remain to be further elucidated.

AKT is an important transcription factor that plays a pivotal role in promoting and maintaining an invasive phenotype by regulating multiple target genes, including $\mathrm{Bcl}-2$, metalloproteinase-9 (MMP-9) and cyclin D1 (16). Notably, previous research $(17,18)$ has also shown that AKT phosphorylates ILK and mediates its nucleocytoplasmic shuttling and functions in the nucleus. Cyclin D1 is the target of AKT, which has also been implicated in glioma (19-21). In the present study, we demonstrated that cyclin D1 in the ILK-knockdown cells was markedly decreased compared with the level in the control cells. We also demonstrated that proliferation of human glioma cells in response to cyclin D1 were inhibited upon inhibition of ILK activity or expression in vitro. Finally, we also showed that inhibition of ILK resulted in a statistically significant suppression of tumor growth in a mouse xenograft model of U251 tumor growth in nude mice. These data suggest that ILK may be considered as an inhibitor for the suppression of tumor growth.

In conclusion, the results of the present study revealed that ILK knockdown reduced glioma cell migration and invasion at least partly through upregulation of E-cadherin and suppressed glioma cell growth through downregulation of cyclin D1. ILK plays a pivotal role in apoptosis, proliferation, invasion and migration. Our results suggest that ILK may serve as a promising therapeutic target for glioma.

\section{Acknowledgements}

The present study was funded by the Natural Science Foundation of Hebei Province (no. 2012201136) and the Medical Science Special Foundation of Hebei University (no. 2012A2004).

\section{References}

1. Lu Y, Chopp M, Zheng X, Katakowski M, Buller B and Jiang F: MiR-145 reduces ADAM17 expression and inhibits in vitro migration and invasion of glioma cells. Oncol Rep 29: 67-72, 2013.

2. DeAngelis LM: Brain tumors. N Engl J Med 344: 114-123, 2001.

3. Van Meir EG, Hadjipanayis CG, Norden AD, Shu HK, Wen PY and Olson JJ: Exciting new advances in neuro-oncology: The avenue to a cure for malignant glioma. CA Cancer J Clin 60: 166-193, 2010.

4. Stupp R and Roila F; ESMO Guidelines Working Group: Malignant glioma: ESMO clinical recommendations for diagnosis, treatment and follow-up. Ann Oncol 20 (Suppl 4): 126-128, 2009.

5. Che H, Song J, Guo S, Wang W and Gao G: Inhibition of xenograft human glioma tumor growth by lentivirus-mediated gene transfer of alphastatin. Oncol Rep 29: 1101-1107, 2013.

6. Delcommenne M, Tan C, Gray V, Rue L, Woodgett J and Dedhar S: Phosphoinositide-3-OH kinase-dependent regulation of glycogen synthase kinase 3 and protein kinase B/AKT by the integrin-linked kinase. Proc Natl Acad Sci USA 95: 11211-11216, 1998.

7. Peroukides S, Bravou V, Varakis J, Alexopoulos A, Kalofonos H and Papadaki H: ILK overexpression in human hepatocellular carcinoma and liver cirrhosis correlates with activation of Akt. Oncol Rep 20: 1337-1344, 2008. 
8. Yan Z, Yin H, Wang R, Wu D, Sun W, Liu B and Su Q: Overexpression of integrin-linked kinase (ILK) promotes migration and invasion of colorectal cancer cells by inducing epithelial-mesenchymal transition via NF- $\mathrm{BB}$ signaling. Acta Histochem 116: 527-533, 2014

9. Zhao D, Tang XF, Yang K, Liu JY and Ma XR: Over-expression of integrin-linked kinase correlates with aberrant expression of Snail, E-cadherin and $\mathrm{N}$-cadherin in oral squamous cell carcinoma: Implications in tumor progression and metastasis. Clin Exp Metastasis 29: 957-969, 2012.

10. Hannigan GE, McDonald PC, Walsh MP and Dedhar S: Integrinlinked kinase: Not so ‘pseudo' after all. Oncogene 30: 4375-4385, 2011.

11. Mao Q, Lin C, Gao J, Liang X, Gao W, Shen L, Kang L and $\mathrm{Xu}$ B: Mesenchymal stem cells overexpressing integrin-linked kinase attenuate left ventricular remodeling and improve cardiac function after myocardial infarction. Mol Cell Biochem 397: 203-214, 2014.

12. Alberton CL, Kanitz AC, Pinto SS, Antunes AH, Finatto P, Cadore EL and Kruel LF: Determining the anaerobic threshold in water aerobic exercises: A comparison between the heart rate deflection point and the ventilatory method. J Sports Med Phys Fitness 53: 358-367, 2013.

13. Serrano I, McDonald PC, Lock F, Muller WJ and Dedhar S: Inactivation of the Hippo tumour suppressor pathway by integrinlinked kinase. Nat Commun 4: 2976, 2013.

14. Luo L, Liu H, Dong Z, Sun L, Peng Y and Liu F: Small interfering RNA targeting ILK inhibits EMT in human peritoneal mesothelial cells through phosphorylation of GSK-3 $\beta$. Mol Med Rep 10: 137-144, 2014.
15. Yu J, Shi R, Zhang D, Wang E and Qiu X: Expression of integrin-linked kinase in lung squamous cell carcinoma and adenocarcinoma: Correlation with E-cadherin expression, tumor microvessel density and clinical outcome. Virchows Arch 458: 99-107, 2011.

16. Min HS, Choe G, Kim SW, Park YJ, Park J, Youn YK, Park SH, Cho BY and Park SY: S100A4 expression is associated with lymph node metastasis in papillary microcarcinoma of the thyroid. Mod Pathol 21: 748-755, 2008.

17. Robertson BW and Chellaiah MA: Osteopontin induces betacatenin signaling through activation of Akt in prostate cancer cells. Exp Cell Res 316: 1-11, 2010.

18. Plante I, Charbonneau M and Cyr DG: Activation of the integrinlinked kinase pathway downregulates hepatic connexin 32 via nuclear Akt. Carcinogenesis 27: 1923-1929, 2006.

19. Rathod SS, Rani SB, Khan M, Muzumdar D and Shiras A: Tumor suppressive miRNA-34a suppresses cell proliferation and tumor growth of glioma stem cells by targeting Akt and Wnt signaling pathways. FEBS Open Bio 4: 485-495, 2014.

20. Zong H, Cao L, Ma C, Zhao J, Ming X, Shang M and Xu H: Association between the G870A polymorphism of cyclin D1 gene and glioma risk. Tumour Biol 35: 8095-8101, 2014.

21. Zhou CX and Gao Y: Aberrant expression of beta-catenin, Pin1 and cyclin D1 in salivary adenoid cystic carcinoma: Relation to tumor proliferation and metastasis. Oncol Rep 16: 505-511, 2006. 